\title{
Time-dependent Response of Eyelid Height with a Single Drop of 2.5\% Phenylephrine in Korean Ptotic Patients
}

\author{
Moon Kyeong Shin, Nam Yeong Kim, Hee Bae Ahn \\ Department of Ophthalmology, Dong-A University College of Medicine, Busan, Korea
}

Purpose: We sought to investigate the temporal changes of eyelid height after phenylephrine instillation in Korean patients with and without ptosis to determine the time points of the first and maximum reactions.

Methods: The phenylephrine test was performed on 16 eyes of 12 ptotic patients (group I) and 24 eyes of 12 normal control subjects (group II) in our hospital between September 2017 and March 2018. One drop of 2.5\% phenylephrine was instilled and the marginal reflex distance 1 (MRD1) was measured at 15 seconds before instillation and the following time points after instillation: at 15-second intervals for the initial 5 minutes and at 5-minute intervals until a total of 20 minutes was reached (i.e., at 10,15 , and 20 minutes).

Results: In group I patients, the first reaction appeared at 5 minutes $(p=0.034)$, while the maximum eyelid height after the first reaction was reached at 15 minutes $(p=0.025)$ and was maintained until 20 minutes. In group II subjects, the first reaction appeared at 5 minutes $(p=0.034)$, while the maximum eyelid height was reached at 10 minutes $(p=0.015)$ and was maintained until 20 minutes. There was no significant difference in the response of eyelid height based on time $(p=0.122)$ between the two groups.

Conclusions: Our analysis of phenylephrine test results in Korean ptotic patients revealed a significant increase occurred in the eyelid height after 5 minutes; meanwhile, the maximum eyelid height was reached at 15 minutes and was maintained until 20 minutes after instillation.

Key Words: Marginal reflex distance, Muller muscle, Phenylephrine test, Ptosis

Ptosis is a condition wherein the upper eyelid margin sits abnormally low when the patient looks forward, caused by the narrowing of the lid fissure [1]. Ptosis can be classified as mild, moderate, or severe, with the decision contingent upon the difference in the palpebral fissure and the func-

Received: February 21, 2020 Final revision: April 13, 2020

Accepted: April 20, 2020

Corresponding Author: Hee Bae Ahn, MD, PhD. Department of Ophthalmology, Dong-A University College of Medicine, 26 Daesingongwon-ro, Seo-gu, Busan 49201, Korea. Tel: 82-51-240-5228, Fax: 82-51254-1987, E-mail: hbahn@dau.ac.kr tioning of the levator palpebral muscle $[2,3]$. The use of phenylephrine, a sympathetic nerve agent, is reportedly helpful in determining the functioning of Müller's muscle, which is under the control of the sympathetic nerve system, and making a decision regarding the pursuit of conjunctival Müller muscle resection (CMMR) [4,5].

Currently, phenylephrine tests are performed by instilling $2.5 \%$ or $10 \%$ phenylephrine and watching for the improvement of ptosis. The timing of the evaluation of the response to phenylephrine varies among studies; in most, the degree of resolution of ptosis was measured after two 
to 10 minutes to decide on the extent of the CMMR [6-8].

Studies measuring and analyzing the formative changes of Korean eyelids have reported that the marginal reflex distance 1 (MRD1) decreases with age [9]. A measurement of MRD1 is commonly used to determine the extent of ptosis. In Korea, phenylephrine tests are often used when making a decision about surgical treatment in ptotic patients; however, the optimal timing of the reaction evaluation is not clearly chosen. The purpose of this study was therefore to investigate temporal changes in the eyelid height at various time points after phenylephrine instillation in Koreans to determine when the first and the maximum reactions to the drug occur.

\section{Materials and Methods}

This study was conducted involving a ptotic patient group of 12 patients ( $\mathrm{n}=16$ eyes) diagnosed with unilateral or bilateral ptosis between September 2017 and March 2018 (group I) and a second group (group II) involving 12 healthy subjects $(n=24$ eyes) as the control group. All participants' medical records were retrospectively analyzed. The criterion for ptosis was defined as either an MRD1 of less than $2.5 \mathrm{~mm}$ or $2 \mathrm{~mm}$ or greater difference in MRD1 between the two eyes. Patients with a history of eyelid surgery or ocular disease that had an effect on the eyelid function were excluded. This study was approved by the Dong-A University's institutional review board for clinical research (DAUHIRB-19-010) and the study protocol followed the guidelines of the Declaration of Helsinki. Participants were recruited from the Department of Ophthalmology, Dong-A University Hospital. Informed consent was waived due to the retrospective nature of the study.

A drop of $2.5 \%$ phenylephrine hydrochloride $(2.5 \%$; Mydfrin, Alcon, Fort Worth, TX, USA) was instilled to measure changes in MRD1. Photographs were taken at 24 time points, including at 15 seconds before instillation, at 15 -second intervals for the initial 5 minutes after instillation, and then at 5-minute intervals until 20 minutes (i.e., 10,15 , and 20 minutes). MRD1 was measured three times using the ImageJ program (National Institutes of Health, Bethesda, MD, USA) and mean and standard error values were calculated for analysis (Fig. 1).

MRD1 is the distance between the corneal reflections from the edge of the upper eyelid. It was measured by an examiner who instructed subjects to look at the iris of the camera without lifting the chin to take a photograph of the entire face of the standard coronal plane. An increase in MRD1 of $0.5 \mathrm{~mm}$ or higher in the phenylephrine test was considered to suggest a positive response.

The first reaction was defined as the first time a statistically significant increase in MRD1 was observed during the observation period, while the maximum reaction was
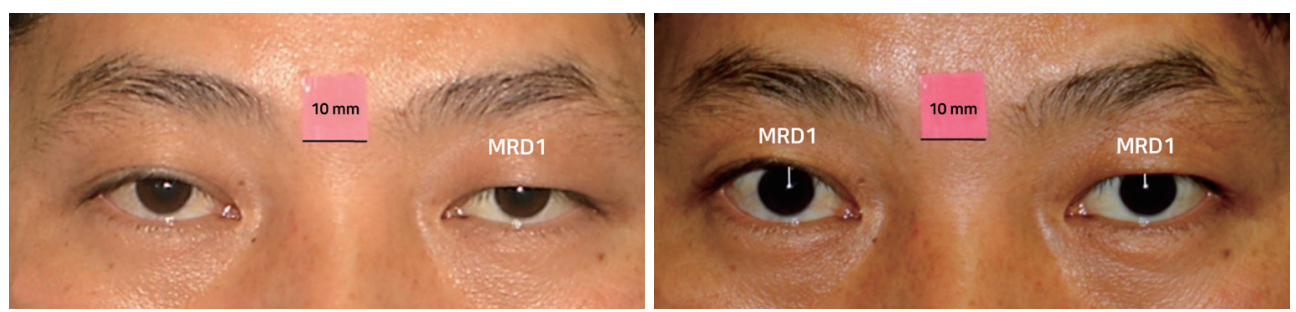

Fig. 1. Changes in marginal reflex distance 1 (MRD1). (A) Pretest and (B) posttest MRD1 are measured by image J program. Written informed consent was obtained from the patient.

Table 1. Changes in MRD1 in group I (ptotic group)

\begin{tabular}{lcccccc}
\hline & Pretest & $2 \min$ & $5 \min$ & $10 \min$ & $15 \min$ & $20 \min$ \\
\hline MRD1 & $1.17 \pm 0.34$ & $1.4 \pm 0.39$ & $1.97 \pm 0.45$ & $2.38 \pm 0.40$ & $2.53 \pm 0.40$ & $2.53 \pm 0.40$ \\
$p$-value & & 1.000 & $0.034^{*}$ & $<0.001^{*}$ & $<0.001^{*}$ & $<0.001^{*}$ \\
\hline
\end{tabular}

Values are presented as the mean \pm standard error of the mean.

MRD1 = marginal reflex distance 1 .

"Statistical significance is $p<0.05$ (repeated-measures analysis of variance). 
defined as the time point at which the maximum eyelid height was reached, which was statistically significantly higher than the height at the first reaction.

Statistical analysis was performed using the SPSS ver. 12.0 (SPSS Inc., Chicago, IL, USA). A repeated-measures analysis of variance was performed and a $p$-value less than 0.05 was considered to indicate statistical significance. Additionally, Bonferroni correction was conducted to correct for errors that may have occurred in multiple comparisons.

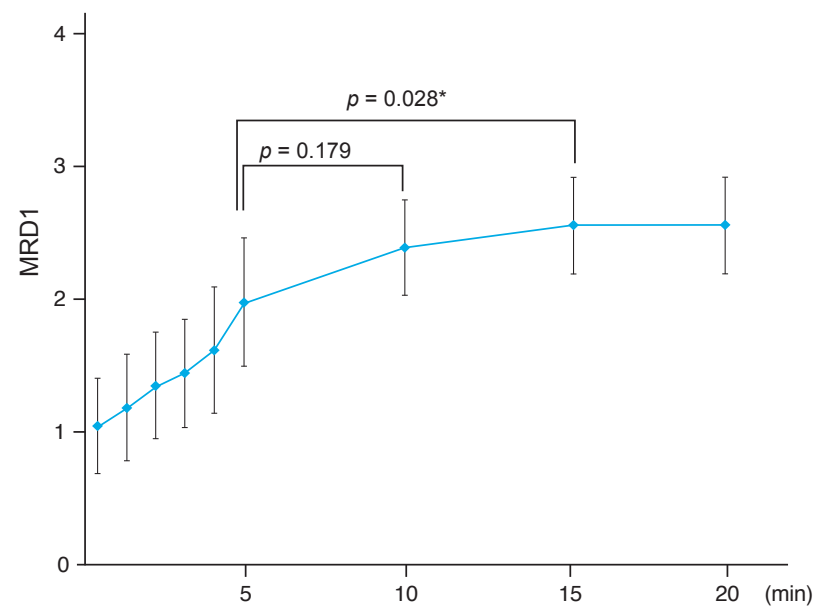

Fig. 2. The changes of marginal reflex distance 1 (MRD1) in group I (ptotic group). "Statistical significance is $p<0.05$ (repeated-measures analysis of variance).

\section{Results}

A total of 24 participants ( $n=40$ eyes) were enrolled, including 13 females and 11 males, whose mean age was 46.9 \pm 18.2 years. Of the 40 eyes assessed, 38 tested positive during the phenylephrine test, thus excepting two eyes of two people (one eye of a ptotic patient and one eye of a normal subject). However, measurements for all patients regardless of the phenylephrine test results were used in the statistical analysis.

In group I, the mean preoperative MRD1 was $1.17 \pm 0.34$ $\mathrm{mm}$ and the mean postoperative MRD1 was $1.97 \pm 0.45$ $\mathrm{mm}$ at 5 minutes after phenylephrine instillation, suggesting a statistically significant increase had occurred by the time of the first reaction $(p=0.034)$. Meanwhile, at 15 minutes after instillation, the mean MRD1 had increased to $2.53 \pm 0.40 \mathrm{~mm}(p \leq 0.001)$ and remained unchanged thereafter until 20 minutes (Table 1). After the first reaction at 5 minutes, a statistically significant maximum reaction $(p=0.028)$ was first observed at 15 minutes (Table 2 and Fig 2).

The mean preoperative MRD1 of group II subjects was $3.04 \pm 0.13 \mathrm{~mm}$, and their mean postoperative MRD1 was $3.54 \pm 016 \mathrm{~mm}$ at 5 minutes after phenylephrine instillation, suggesting a statistically significant increase had occurred by the time of the first reaction $(p=0.034)$. At 15 minutes after instillation, the mean MRD1 had increased

Table 2. Changes in marginal reflex distance 1 in group I (ptotic group) after early reaction

\begin{tabular}{|c|c|c|c|c|c|}
\hline \multirow{2}{*}{ Dependent variable } & & \multirow{2}{*}{ Mean difference } & \multirow{2}{*}{ Significance } & \multicolumn{2}{|c|}{$95 \%$ confidence interval ${ }^{*}$} \\
\hline & & & & Lower bound & Upper bound \\
\hline \multirow[t]{3}{*}{$5 \min$} & $10 \mathrm{~min}$ & -0.406 & 0.179 & -0.909 & 0.096 \\
\hline & $15 \mathrm{~min}$ & $-0.563^{*}$ & $0.028^{\dagger}$ & -1.079 & -0.046 \\
\hline & $20 \mathrm{~min}$ & $-0.566^{*}$ & $0.026^{\dagger}$ & -1.062 & -0.050 \\
\hline
\end{tabular}

*Adjustment for multiple comparisons: Bonferroni; ’Statistical significance is $p<0.05$ (repeated-measures analysis of variance).

Table 3. Changes in MRD1 in group II (normal group)

\begin{tabular}{lcccccc}
\hline & Pretest & $2 \mathrm{~min}$ & $5 \mathrm{~min}$ & $10 \mathrm{~min}$ & $15 \min$ & $20 \mathrm{~min}$ \\
\hline MRD1 & $3.04 \pm 0.13$ & $3.09 \pm 0.14$ & $3.54 \pm 0.16$ & $3.79 \pm 0.13$ & $3.85 \pm 0.12$ & $3.85 \pm 0.12$ \\
$p$-value & & 1.000 & $0.034^{*}$ & $<0.001^{*}$ & $<0.001^{*}$ & $<0.001^{*}$ \\
\hline
\end{tabular}

Values are presented as the mean \pm standard error of the mean.

MRD1 = marginal reflex distance 1 .

${ }^{*}$ Statistical significance is $p<0.05$ (repeated-measures analysis of variance). 


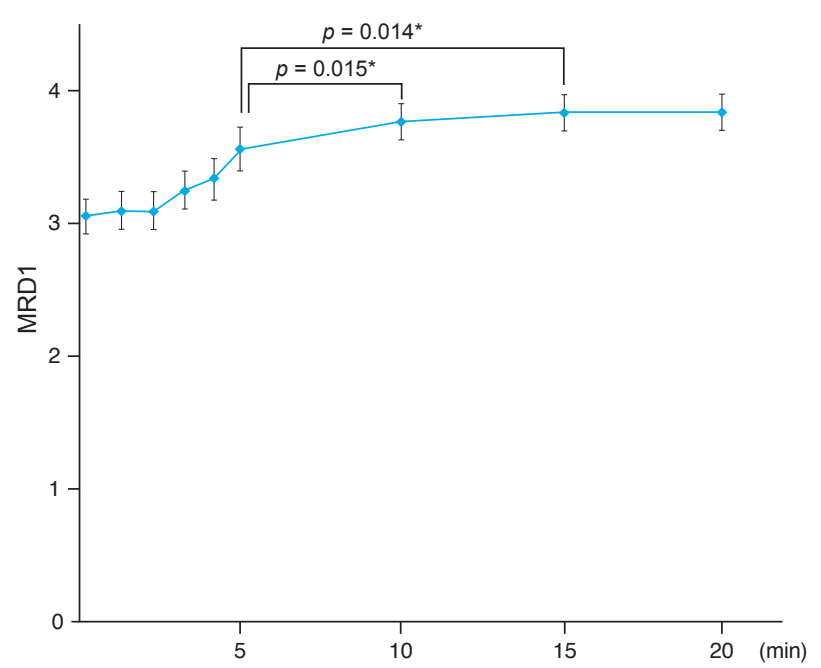

Fig. 3. The changes of marginal reflex distance 1 (MRD1) in group II (normal group). "Statistical significance is $p<0.05$ (repeated-measures analysis of variance).

to $3.85 \pm 0.12 \mathrm{~mm}(p \leq 0.001)$ and remained unchanged thereafter until 20 minutes (Table 3). After the first reaction at 5 minutes, a statistically significant maximum reaction was first observed at 10 minutes $(p=0.015)$ (Table 4 and Fig 3).

Overall, these results reveal that the time to reach the maximum eyelid height occurred within the 15 minutes after instillation in the patient group and within 10 minutes after instillation in the normal control group, with the time difference between the two being statistically significant $(p=0.122)$.

\section{Discussion}

The phenylephrine test is used to assess the function of Müller's muscle in ptotic patients and to help discern whether CMMR must be performed. Conventionally, the phenylephrine test is performed by instilling $2.5 \%$ or $10 \%$ phenylephrine and acquiring MDR1 measurements in 3 to 5 minutes to determine any resolution in ptosis. Studies vary in the time after phenylephrine instillation at which MDR1 measurements are acquired to evaluate the patient's reaction to the drug. Ramesh and Mancini [6] reported that the maximum reaction was reached within two minutes from instilling a drop of $2.5 \%$ phenylephrine, while Grace Lee et al. [7] reported this time to be 5 minutes after instilling a drop of $10 \%$ phenylephrine. Meanwhile, Ben et al. [8] measured the degree of ptosis improvement at 10 minutes after instilling a drop of $10 \%$ phenylephrine to determine the extent to which CMMR needs to be performed.

In light of this discrepancy, in the present study, we decided to investigate the timing of the first reaction and the maximum reaction by analyzing changes in eyelid height over time after phenylephrine instillation in Korean patients. The phenylephrine test results of ptotic patients revealed that the eyelid height experienced a significant increase at 5 minutes after instillation and peaked at 15 minutes, suggesting 5 minutes to be the time of the first reaction and 15 minutes to be that of the maximum reaction. This is a different result from previous studies, where the timing of the maximum reaction was reported to be less than 10 minutes after instillation.

The slower response to phenylephrine testing in Western populations relative to Koreans is probably because the latter group tends to present larger amounts of adipose tissues in their upper eyelids and the anatomical structures of Müller's muscle differs between the two populations. In particular, Müller's muscle is controlled by sympathetic nerves and serves as the involuntary ptosis crutch of the upper eyelid. Müller's muscle begins at the aponeurosis of the levator palpebrae superioris muscle and is attached to the superior tarsal border; the posterior surface of Müller's

Table 4. Changes in marginal reflex distance 1 in group II (normal group) after early reaction

\begin{tabular}{lccccc}
\hline \multirow{2}{*}{ Dependentvariable } & & \multirow{2}{*}{ Mean difference } & Significance & \multicolumn{2}{c}{$95 \%$ confidence interval $^{*}$} \\
\hline $5 \mathrm{~min}$ & $10 \mathrm{~min}$ & $-0.252^{\dagger}$ & $0.015^{\dagger}$ & -0.909 & Lower bound \\
& $15 \mathrm{~min}$ & $-0.313^{\dagger}$ & $0.014^{\dagger}$ & -1.079 & -0.046 \\
& $20 \mathrm{~min}$ & $-0.313^{\dagger}$ & $0.013^{\dagger}$ & -1.062 & -0.050 \\
\hline
\end{tabular}

${ }^{*}$ Adjustment for multiple comparisons: Bonferroni; ${ }^{\dagger}$ Statistical significance is $p<0.05$ (repeated-measures analysis of variance). 
muscle is firmly attached to the conjunctiva, while its anterior surface is loosely attached to the aponeurosis of the levator palpebrae superioris muscle [10]. A previous study on the anatomical structure of Müller's muscle in the levator palpebrae superioris muscle reported its anatomy in Koreans to be different from that in Westerners as the former did not show that Müller's muscle was directly attached to the superior tarsal border, unlike in the latter [11]. Therefore, phenylephrine testing in Koreans requires a response time of 15 minutes or longer, which is longer than previously suggested.

Limitations of this study include that it did not assess how long the effects of phenylephrine last and when they wear off. Additional research is needed to determine this information. In addition, considering the study's small sample, research with larger sample sizes should be conducted in the future. Additional research is also needed to compare the effects of phenylephrine between eyes with varying degrees of ptosis. Finally, further investigation is warranted concerning different types of eye drops including $2.5 \%$ phenylephrine hydrochloride, $10 \%$ phenylephrine hydrochloride, and $0.5 \%$ iopidine (Akorn, Fort Worth, TX, USA).

In conclusion, measurements after phenylephrine instillation in Korean ptotic patients should be taken after allowing for a sufficient response development time of 15 minutes. It is believed that accurate phenylephrine testing is instrumental in determining the best operation protocol, such as the extent of CMMR that needs to be performed.

\section{Conflict of Interest}

No potential conflict of interest relevant to this article was reported.

\section{Acknowledgements}

This work was supported by the Dong-A University research fund.

\section{References}

1. Finsterer J. Ptosis: causes, presentation, and management. Aesthetic Plast Surg 2003;27:193-204.

2. Small RG, Sabates NR, Burrows D. The measurement and definition of ptosis. Ophthalmic Plast Reconstr Surg 1989;5:1715.

3. Kim IS, Choi JB, Rah SH, Lee SY. Classification of ptosis in Korea. J Korean Ophthalmol Soc 2005;46:1262-9.

4. Dresner SC. Further modifications of the Muller's muscle-conjunctival resection procedure for blepharoptosis. Ophthalmic Plast Reconstr Surg 1991;7:114-22.

5. Bae JS, Ha MS, Lee JY, et al. Results of conjunctiva-Muller muscle resection in mild eyelid ptosis. J Korean Ophthalmol Soc 2008;49:1365-70.

6. Ramesh S, Mancini R. Dynamic analysis of Muller's muscle response to phenylephrine. Ophthalmic Plast Reconstr Surg 2016;32:46-8.

7. Grace Lee N, Lin LW, Mehta S, Freitag SK. Response to phenylephrine testing in upper eyelids with ptosis. Digit $J$ Ophthalmol 2015;21:1-12.

8. Ben Simon GJ, Lee S, Schwarcz RM, et al. Muller's muscle-conjunctival resection for correction of upper eyelid ptosis: relationship between phenylephrine testing and the amount of tissue resected with final eyelid position. Arch Facial Plast Surg 2007;9:413-7.

9. Seo HR, Ahn HB. Morphological changes of the eyelid according to age. J Korean Ophthalmol Soc 2009;50:1461-7.

10. Button JJ. Atlas of clinical and surgical orbital anatomy. Philadelphia: WB Saunders; 1994 . p. 113-38.

11. Ahn HB, Oh HC, Roh MS, et al. The study of anatomic relationship between the Muller muscle and the tarsus in Asian upper eyelid. Ophthalmic Plast Reconstr Surg 2010;26:334-8. 